\title{
La memoria de los pueblos en la Colección de Preguntas y Respuestas del Instituto Centroamericano de Extensión de la Cultura (ICECU)
}

\section{People's Memory at the ICECU's Questions and Answers Collection}

Daniel Bonilla Matamoros*

Resumen: La Colección de Preguntas y Respuestas del ICECU constituye una fuente documental privilegiada, única, poco explorada y con una amplia riqueza. En este texto se realiza una descripción del trabajo del ICECU y de su archivo, y se brinda una aproximación a las diferentes temáticas que lo conforman.

Palabras claves: Centroamérica; historia oral; patrimonio documental; vida cotidiana.

Abstract: The ICECU Question and Answer Collection is a privileged, unique, unexplored and broad wealth documentary source. In this text, a description of the work of ICECU and its archive is made, and an approximation to the different themes that comprise it is provided.

Keywords: Central America; Oral History; Documentary Heritage; DailyLlife.

\section{¿Qué es el ICECU?}

$\int$ n San Pedro de Montes de Oca, San José, Costa Rica, existe un acervo documental que, entre sus hojas ya amarillas por el paso del tiempo, refleja la cosmovisión de una región, las preocupaciones e intereses de muchos pueblos, la cotidianidad de muchas personas y otra gran variedad de temáticas. Todo esto y más se encuentra contenido en la Colección de Preguntas y Respuestas del Instituto Centroamericano de Extensión de la Cultura (ICECU). ${ }^{1}$

Fecha de recepción: $28 / 01 / 2020$

* Costarricense. Bachiller en Historia por la Universidad de Costa Rica (UCR), Costa Rica. Colaborador en el Instituto Centroamericano de Extensión de la Cultura (ICECU), San Pedro de Montes de Oca, Costa Rica. Correo electrónico: dbonillamatamoros@gmail.com. 
El ICECU es una institución creada por la Ley 3215 de la Asamblea Legislativa de Costa Rica, del 19 de octubre de 1963. El ICECU es el trabajo y el esfuerzo de la costarricense doña Manuela Tattenbach y el austriaco Roderick Thun, mismos fundadores del libro Almanaque Escuela Para Todos que se publica ininterrumpidamente desde el año 1966.

Durante su vida y en contacto con diferentes poblaciones, Tattenbach y Thun identificaron una necesidad compartida por muchas personas que, por una u otra razón, no formaron parte del sistema educativo formal: el acceso a la información en un lenguaje accesible y comprensible. Se cuenta que día a día, los campesinos tocaban su puerta para hacer diferentes preguntas de temáticas varias. Tattenbach y Thun, con la ayuda de libros, dispositivas, fotografias y demás materiales, se daban a la tarea de evacuar esas preguntas. ${ }^{2}$

A partir de esta y otras experiencias nació la idea de crear un programa de radio -aprovechando el entonces novedoso radio de transistores- donde se explicaran diversas temáticas con un lenguaje fácil de comprender. Así se creó el programa radial Escuela Para Todos — hoy llamado Oigamos la Respuesta-que salió al aire inicialmente solo en Costa Rica el 12 de octubre de 1964, por medio de las antenas de Radio Monumental. En poco tiempo, el programa se empezó a transmitir en otros países de la región centroamericana, y esto continúa en la actualidad más la transmisión por medio de Internet en formato podcast.

Thun y Tattenbach, además identificaron gran escasez en material de lectura accesible y redactado de una manera sencilla. Fue así que crearon el libro Almanaque Escuela Para Todos ${ }^{3}$ cuyo primer número se publicó en el año de 1966.

\section{¿Cómo trabaja el ICECU?}

Volviendo al programa de radio, este cuenta con un formato particular que nos permite explicar la metodología y el trabajo del ICECU. Se trata de un programa radial de preguntas y respuestas. Eso sí, desde un inicio, Tattenbach y Thun dejaron claro que el programa se realizaba no con el fin de transmitir lo que estos consideraban importante, sino para comunicar, por medio de la radio, los temas que las personas oyentes quisieran saber. ${ }^{4}$ De esta manera, se establece un puente de comunicación en el que la persona que envía su pregunta al ICECU, se reconoce parte del proyecto y también protagonista de «....su propio crecimiento cultural, e incluso con la posibilidad de apoyar solidariamente el crecimiento cultural de sus compañeros de aprendizaje...».5

1 Visítese la página Web del ICECU por medio del enlace: https://icecu.org/. Su correo electrónico es: icecu@icecu.org.

2 La Nación, «Todos aprendemos en "Escuela para todos"», 30 de enero de 1975, 15 C.

3 Véase su sitio Web: https://almanaqueept.org/.

4 Julio Suñol Leal, Milagro en la cintura de América: un millón de preguntas y respuestas (San José: UACA, 2002), 31.

5 José Manuel Fajardo Salinas, «Los fundamentos del proyecto "Escuela Para Todos" en el área de Mesoamérica desde una lectura filosófica intercultural» (Memoria para optar al grado de Magíster en Ética Social y Desarrollo Humano, Universidad Alberto Hurtado, Chile, 2007), 71. 
En sus inicios, en los primeros programas de radio se respondieron preguntas de los propios empleados de la institución, pero conforme se contrataron estaciones de radio para difundirlo, y otras prestaron un espacio dentro de su programación, el ICECU empezó a recibir cartas y llamadas telefónicas de oyentes que reportaban la sintonía y realizaban diferentes preguntas de variados temas. De igual manera, se empezaron a recibir consultas provenientes de diferentes lugares de Centroamérica conforme la transmisión del programa cubría más y más territorio en la región. Hoy día, como se mencionó, el programa radial se transmite, en distintas emisoras de toda la región centroamericana, sin interrupción, desde aquel 12 de octubre del año 1964. Además, el formato inicial se mantiene y siguen llegando al ICECU preguntas de una gran diversidad temática, de diferentes lugares, de niños y de adultos, de personas con distinta formación educativa y por diversos medios: desde visitas, cartas, llamadas telefónicas, correo electrónico, Facebook y hasta WhatsApp.

Las preguntas que ingresan al ICECU ${ }^{6}$ se clasifican entre aquellas cuya respuesta se puede tomar del archivo institucional y las que tratan temas que no se han preguntado anteriormente. Después, se redactan las respuestas y luego de un proceso de revisión se envían a las personas que las remitieron. Con estas preguntas se hace el programa radial Oigamos la Respuesta que luego de ser editado se envía a las diferentes radioemisoras que transmiten el programa en distintos lugares de la región. Para responder a estas preguntas se recurre a especialistas de diferentes instituciones, por lo que, como señala Suñol, se establece un contacto entre la persona que pregunta con el mundo académico. ${ }^{7}$

La particularidad de estas respuestas es que deben ser redactadas de manera comprensible. De este principio el lema tanto del ICECU como de la Fundación Escuela Para Todos: «Comprender lo comprensible es un derecho humano». ${ }^{8} \mathrm{Cada}$ pregunta representa un reto pues las personas encargadas de dar respuesta deben «convertirse en maestros de la redacción, capacitados para transformar en material legible y entendible los temas más abstractos, sean estos científicos, literarios, históricos, religiosos, económicos, geográficos o humanos en general». ${ }^{9}$

Sobre lo anterior, el filósofo y educador Guillermo Malavassi menciona que el ICECU enfrenta una lucha con cada una de las consultas que recibe: una lucha contra el idioma, a fin de que la respuesta sea redactada de tal manera que pueda ser entendida por la persona que la remitió y espera la contestación. Malavassi menciona: «La lucha contra lo ininteligible del lenguaje difícil tal vez constituye uno de los mayores esfuerzos del ICECU» ${ }^{10}$ esto debido a que en muchas ocasiones la información disponible se encuentra redactada con

6 También se reciben preguntas de México y además, de centroamericanos que han migrado a los Estados Unidos.

7 Suñol Leal, 41.

8 Frase original del educador alemán Martin Wagenschein (1896-1988).

9 Suñol Leal, 50.

10 Guillermo Malavassi, Comprender lo comprensible. Tomo I (San José: Imprenta Nacional, 1979), 400. 
términos y con conceptos quizás ajenos a muchas personas, por lo que esta se vuelve casi ininteligible. Es por eso que la redacción de dichas respuestas y de los guiones radiofónicos buscan una expresión clara para que pueda ser entendida por todas las personas. ${ }^{11}$

Dicha tarea no resulta fácil: «no es fácil narrar los esfuerzos relativos a "traducir" conceptos, argumentos, deducciones, que sí pueden ser fácilmente comprensibles en ciertos medios de rigurosa formación especializada, sin embargo, de esta manera no serían comprendidos en otros medios humanos con los cuales está en constante comunicación el ICECU». ${ }^{12}$

\section{¿Qué contiene la Colección de Preguntas y Respuestas?}

Esta Colección se conforma por estas preguntas que ingresan al ICECU, y en su conjunto representa una fuente documental de primera mano para el trabajo diario de la institución, pero también un inexplorado corpus documental pues «.... a lo largo de su existencia, de la interrelación entre el ICECU y sus oyentes, se ha ido generando un amplio fondo documental en cuyo fluir circula no solo información, sino también una rica visión de mundo, dudas, certezas, valores y creencias»». ${ }^{13}$

Esta Colección de Preguntas y Respuestas se encuentra dentro de las instalaciones del ICECU, en San Pedro de Montes de Oca, muy cerca de la Universidad de Costa Rica, institución con la que el ICECU mantiene un estrecho vínculo de cooperación, pues sus profesionales contribuyen de manera desinteresada en la actualización de conocimientos sobre diferentes temáticas. Este puente de colaboración permite difundir material de la academia actualizada con una proyección centroamericana.

La Colección de Preguntas y Respuestas puede consultarse mediante cita previa y siguiendo la reglamentación interna de la institución. Esta colección, llamada como el «Archivo del ICECU», constaba para el año 2009 con «más de 240.000 folios los cuales ocupan 190 metros lineales»..$^{14}$ Este acervo cuenta con una amplitud temporal extensa, pues ha sido alimentado ininterrumpidamente durante los 55 años de existencia del ICECU. Y se compone por las preguntas, junto a su correspondiente respuesta, que ha recibido a lo largo de todos esos años el ICECU. La región centroamericana es la que más ha alimentado este archivo, pero también se han recibido, aunque en menor número, consultas de México, Estados Unidos, entre otros países, lo que representa su amplia cobertura espacial. ${ }^{15}$

11 Ibíd., 405 .

12 Ibíd., 406.

13 Instituto Costarricenses de Extensión de la Cultura, Postulación de la Colección de Preguntas y Respuestas del ICECU al Registro Memoria del Mundo de Costa Rica (San José: ICECU, 2009), 3. 
Cada folio de este acopio documental posee la copia textual de la pregunta o del comentario enviado a la institución, junto a la respuesta elaborada por el equipo de redactores del ICECU. ${ }^{16}$ Estas interrogaciones se encuentran organizadas por carpetas, que a la vez están organizadas en ochos grandes grupos temáticos que son:
1. Medicina y salud
2. Plantas y agricultura
3. Zoología
4. Ciencias básicas
5. Arte, educación, deportes
6. Ciencias sociales
7. Religión, filosofía de la vida
8. Comentarios al quehacer institucional del ICECU ${ }^{17}$

De igual manera, estos grupos temáticos se subdividen en diferentes subtemas que se encuentran organizados alfabéticamente. Este orden del archivo «es propio de la institución y obedece a su práctica cotidiana: son las personas que escriben al programa, para hacer preguntas o para compartir su saber, las que han ido creando esta inmensa colección y han generado la gran multiplicidad de tópicos que contiene». ${ }^{18}$

¿Cuál es el perfil de los remitentes? Personas en su mayoría «...sencilla y humilde, y esto se revela por la ortografía, la regular calidad el papel utilizado y la letra, aunque los remitentes muestran profunda sed de conocimientos y un deseo inmenso por encontrar explicaciones a los más diversos fenómenos y acontecimientos presentes, pasados e históricos». ${ }^{19} \mathrm{Si}$ se sigue la descripción de Suñol, las consultas que ingresan al ICECU, entre comentarios y otros mensajes, tienen una variedad temática enorme. Suñol menciona que «Las preguntas tienen relación con los países y sus costumbres, la geografía, la astronomía, las personalidades mundiales, las ciencias y las letras, la historia, la medicina, el sida, la gastritis... $\rangle^{20} \mathrm{y}$ muchísimos temas más.

Las cartas, y hoy día también mensajes de WhatsApp, correo electrónico y más, son, dice Malavassi: cortas y largas. Estas cartas que por mucho tiempo fueron la vía principal de comunicación entre el ICECU y los oyentes del programa radial, dice Malavassi, en ocasiones llegaban con «... una sola pregunta y hasta de varias decenas de ellas. Algunas vienen mecanografiadas, otras escritas en garabatos de muy difícil lectura; unas vienen en español, otras en idiomas que

16 Ibíd., 9.

17 Ibíd., 14.

18 Ibíd. Cabe mencionar que este archivo se encuentra en un proceso inicial de digitalización.

19 Suñol Leal, 51.

20 Ibíd. 
resulta difícil descifrar. Las hay en papel fino y las que vienen en papel de envolver, detrás de papel usado, en cualquier superficie en que se pueda escribir... $\rangle^{21}$ como las servilletas.

Los temas tratados por las personas remitentes son amplios, lo que muestra, según Malavassi, «...una expresión tan amplia del multiforme espíritu humano a través de los miles de preguntas que han llegado, que solo cabe considerar lo extraordinaria que es la gana de saber y la necesidad de comunicarse, modos de realizar el ser humano su naturaleza». ${ }^{22}$

Este acervo documental es testimonio de primera mano de valoraciones, ideas, opiniones, identidades y mucho más, lo que manifiesta una riqueza en su contenido: «....reflejando intereses que van de lo personal o local hasta lo general y universal; cuestiones relativas a acontecimientos presentes o del más lejano pasado; a lugares habituales o remotos. Asimismo, tratan desde aspectos pragmáticos o utilitarios, hasta cavilaciones de la más sublime trascendencia». ${ }^{23}$

Por estos y otros motivos ${ }^{24}$ la Colección de Preguntas y Respuestas del ICECU recibió el certificado por parte de la UNESCO en Costa Rica como parte en el Registro Nacional de Memoria del Mundo según acuerdo n. ${ }^{\circ}$ 1, de la sesión 4-2009, el 18 de setiembre de 2009. Además, en diciembre del mismo año esta Colección se incorporó al Registro Memoria del Mundo de América Latina y el Caribe por parte del Comité Regional para América Latina y el Caribe MOWLAC por su acróstico en inglés-. El Programa Memoria del Mundo tiene como objetivo el conservar la memoria colectiva de la humanidad por medio de la preservación de diferente patrimonio documental..$^{25}$

Esta Colección de Preguntas y Respuestas no acaba, pues cada día ingresan al ICECU nuevas interrogantes con una gran diversidad temática que responde al contexto particular de cada persona, pero también al contexto local, nacional e incluso mundial. Estas interrogantes además mantienen el archivo en una profunda y constante actualización. La Colección, como ya se ha mencionado, representa, entre muchas otras aristas, el sentir de diferentes personas, de diferentes pueblos centroamericanos en distintos contextos. Ha sido alimentado especialmente por personas sencillas, pero con una capacidad de observación y

21 Guillermo Malavassi, Comprender lo comprensible. Tomo II (San José: Imprenta Nacional, 1979), 75.

22 Ibíd, 76.

23 Instituto Costarricenses de Extensión de la Cultura, 14-15.

24 En la postulación de la Colección como Registro Memoria del Mundo se mencionan algunos aspectos que hacen único a este acervo documental, citamos varios de ellos: a) colección de carácter regional, única en su tipo en Centroamérica y el Caribe e incluso en América Latina, b) colección que se nutre del diálogo permanente con los oyentes y a lo largo de más de 50 años de trabajo ininterrumpido, c) una base intercultural representada en la relación de preguntas y respuestas y que da voz a poblaciones silenciadas, d) amplia cobertura temporal y espacial en la procedencia de la correspondencia recibida, tomado de: Instituto Costarricenses de Extensión de la Cultura, 15-16.

25 Tomado de «Programa Memoria del Mundo (MoW): Preservando el patrimonio documental», http://www. unesco.org/new/es/santiago/communication-information/. 
un deseo de conocimiento inquebrantable. Sus folios dejan entrever el pensar de muchas personas, distintas mentalidades, representaciones e ideas que se pueden leer en hojas ya amarillas por el pasar del tiempo.

Para finalizar, transcribimos algunas de las preguntas que se encuentran en esta Colección de Preguntas y Respuestas del ICECU, a fin de ilustrar la gama de consultas que se reciben, advirtiendo que la muestra se queda corta con la amplitud temática que posee este acervo: ${ }^{26}$

- Nicaragua, Masaya, año 1966: «¿dónde está el viento cuando no sopla?».

- Guatemala, Chirijquiac, año 1967: «quiero saber si es cierto que en el remolino que hay un animal está por dentro el remolino».

- $\quad$ El Salvador, Cabaña, año 1968: «deseo saber ¿si todas las luces de las estrellas que vemos se consideran que su luz ha llegado a la Tierra? Pues según mi opinión, yo creo que solamente la luz del Sol y de la Luna son las únicas que llegan a la Tierra, porque la iluminan las luces de las estrellas. No creo que lleguen hasta la Tierra, porque cuando no hay luna es noche oscura. Pero ustedes me dirán si esto es así o no».

- Nicaragua, Managua, año 1970: «ipor qué le pusieron el nombre el Vaticano a ese Estado?».

- Guatemala, año 1972: «¿cómo están prendidas las estrellas y de dónde sale la oscuridad?».

- Honduras, Aldea Monte Rey, año 1973: «¿será posible que al morir uno y lo entierran, allí está el espíritu con uno hasta el día del juicio? Pes yo no creo eso, quisiera que me dijeran cómo será».

- $\quad$ Nicaragua, Chinandega, año 1974: «¿Por qué el huevo de pato dilata más en nacer que el huevo de gallina? Porque yo he echado huevos de gallina con huevos de patos, pero los de patos los hecho primero, y los de gallina hasta después de 8 días».

- $\quad$ Costa Rica, Aranjuez de Puntarenas, año 1974: «nos cuentan que los zopilotes tienen una mosca debajo del ala y que esa mosca sale a buscarle las mortandades al zopilote cuando encuentra comida corren a avisarle a los zopilotes. Yo quiero que me saquen de dudas».

- Honduras, Tegucigalpa, año 1977: «¿de qué lengua proviene la palabra Dios y Diablo? ¿Qué significa en toda su magnitud de la palabra?».

- Honduras, San Pedro Sula, año 1977: «ies cierto que las personas que mueren por suicidio o homicidio andan errantes porque nuestro Señor no los recibe?».

26 No se transcribe el nombre de la persona que envió la pregunta. 
- Guatemala, año 1979: «ipor qué y cómo fue que Guatemala perdió los territorios de Chiapas y Soconusco?».

- $\quad$ Panamá, Chiriquí, año 1979: «deseo que me digan si es cierto que una víbora puede mamar de las tetas de una vaca, pues mi abuelo me contó una historia que él pudo apreciar donde un animal de estos le causó la muerte a un ternero porque lo dejaba sin leche. Y si es cierto que esto sucede ¿cómo es que puede mamar y al agarrar la teta no morderla para no infiltrarle el veneno al animal y causarle la muerte».

- $\quad$ El Salvador, Coatepeque, Departamento de Santa Ana, año 1983: «quisiera saber cómo hacen los gorgojos para meterse dentro del grano del frijol. ¿O será que ahí nacen? Porque yo tengo unos sacos de frijol que se me están picando, porque encuentro uno frijoles que nos están picados y el gorgojo está dentro del grano. ¿Cómo es eso?».

- México, Veracruz, año 1983: «¿qué fue el motivo de la guerra de Guatemala? ¿Por qué ese gobierno no tiene lástima de la gente civil? ¿Cuánto irá a durar esa guerra y quién la irá a ganar?».

- Guatemala, Mixco, año 1984: «ipor qué en las tierras del oriente de Guatemala no existen personas de origen nativas y no hay ningún rasgo de que hayan habitado tribus por esas regiones y la gente es diferente a la del occidente? Se cree que los mayas habitaron las pares altas de Guatemala ¿será posible que hayan habitado en el oriente por el único lugar que tiene rasgos es Izabal con las ruinas de Quirigua y las demás regiones, si todo el oriente es grande?».

- $\quad$ El Salvador, La Libertad, año 1984: «¿por qué se enamoran los humanos? ¿A qué se debe la atracción de cada uno?».

- Guatemala, Chimaltenango, año 1985: «Quiero saber de la historia revolucionaria de 1944 y quiero saber qué pasó con el último gobierno de la revolución de Jacobo Arbenz Guzmán, después del golpe de estado que fue preparado por los Estados Unidos y cuál fue la razón del derrocamiento y quiénes y cómo le dieron muerte al gobierno golpista Carlos Castillo Armas».

- $\quad$ Panamá, Provincia de Coclé, año 1986: «¿creen ustedes que la búsqueda de un Dios supremo en algunas religiones monoteístas es igual a la búsqueda que hacen los politeístas en varios dioses? ¿Por qué ha habido unos pueblos monoteístas y otros politeístas?».

- México, Chiapas, año 1990: «si la Tierra está sentada en agua ¿el agua sobre qué está?».

- $\quad$ Nicaragua, Zelaya, año 1991: «a mí me parece que estando en el espacio a uno se le aparenta estar con la cabeza para arriba y luego sin moverse 
se le aparenta estar con la cabeza para abajo. A mí me parece que estando en el espacio donde no hay astros muy cerca de uno, uno dice: para acá es arriba, luego dice ¡a no! si para acá es arriba y por último no sabe para dónde es para abajo ni arriba, porque para todos lados ve astros, a uno no se le pone el Sol, porque no está en la Tierra estando en el espacio uno ve que no hay tiempo, aunque sé que todos los astro giran en el espacio. Es así ¿no?».

- Guatemala, Huehuetenango, año 1992: «¿Por qué algunas ocasiones algunas gallinas cantan como gallo? Dicen que cuando una gallina canta como gallo, alguien de la familia de la casa se va a morir. ¿Será cierto esto?».

- Guatemala, Guastatoya, año 1993: «quisiera que me sacaran de la siguiente duda. Dicen que en cada cerro hay una serpiente y que cuando ésta se mueve retumba el cerro. Aquí en San Antonio, hay un cerro y cuentan que hace años vinieron unos señores y observaron en una laguna que hay enterrada en el cerro a la serpiente, y que le comenzaban a salir los cachitos. Cuando la vieron la laguna estaba llena, varias personas de aquí cuentan eso y dicen que es cierto, y aseguran que la cola está en el Departamento de El Progreso y la cabeza aquí. Quiero que me digan si es verdad o no».

- Costa Rica, Puntarenas, Golfito, año 1993: «quiero saber si en el mundo en algún tiempo y en algún país del mundo ha habido una huelga de trabajadores que sea declarada legal y que haya sido ganada por los trabajadores».

- Honduras, Choluteca, año 1995: «QQuién es Dios? ¿Dónde está? ¿Cuáles son sus hijos?».

- $\quad$ Nicaragua, Nueva Segovia, año 2004: «quiero que me manden por escrito todo lo que pido a continuación ¿cómo es el globo terráqueo? ¿Cómo está la Tierra, en qué está detenida y qué sigue después? ¿Qué tan largo está el cielo de la Tierra?».

- $\quad$ El Salvador, La Paz, año 2014: «¿cuál es el libro más antiguo de nuestro continente?».

- Costa Rica, Cartago, año 2016: «deseo saber ¿cuándo y cómo llegó la fotografía a Centroamérica?».

- $\quad$ El Salvador, La Libertad, 2018: «¿qué es la política? ¿Por qué los jóvenes dicen que son apolíticos? ¿En qué consiste la real politik?».

- $\quad$ El Salvador, San Salvador, año 2019: «¿en qué idioma fue escrito el "popolbu" y cómo eran las letras en ese entonces?». 


\section{Bibliografía}

«Programa Memoria del Mundo (MoW): Preservando el patrimonio documental». http://www.unesco.org/new/es/santiago/communication-information/ memory-of-the-world-programme-preservation-of-documentary-heritage/.

Fajardo Salinas, José Manuel. «Los fundamentos del proyecto "Escuela Para Todos" en el área de Mesoamérica desde una lectura filosófica intercultural». Memoria para optar al grado de Magíster en Ética Social y Desarrollo Humano, Universidad Alberto Hurtado, Chile, 2007.

Instituto Costarricense de Extensión de la Cultura. Postulación de la Colección de Preguntas y Respuestas del ICECU al Registro Memoria del Mundo de Costa Rica. San José: ICECU, 2009.

La Nación. "Todos aprendemos en "Escuela para todos"», 30 de enero de 1975, 15 C.

Malavassi, Guillermo. Comprender lo comprensible. Tomos I-II. San José: Imprenta Nacional, 1979).

Suñol Leal, Julio. Milagro en la cintura de América: un millón de preguntas y respuestas. San José: UACA, 2002. 